
\title{
AC Losses for the Prototype Cable-in-Conduit Conductors
for NET
}

\author{
P. Bruzzone1, L. Bottura1, J. Eikelboom ${ }^{2}$, A.J.M. Roovers 3
}

1 The NET Team

c/o Max-Planck-Institut für Plasmaphysik, Boltzmannstr. 2, D - 8046 Garching bei München,

2ECN Petten, P.B.1, NL - 1755 ZG Petten

${ }^{3}$ University of Twente, P.O. Box 217, NL - 7500 AE Enschede

Abstract----The AC losses requirement for the superconducting coils of the Next European Torus (NET) project are discussed on the basis of the pulsed field scenario and plasma disruptions. The AC losses test results on the prototype $40 \mathrm{kA}$ cable-in-conduit superconductors developed for NET are presented: the performance achieved is compared with the target. Some recommendations are made for work to be done.

\section{INTRODUCTION}

In order to control and stabilize the plasma current of NET [1] (Next European Torus) the fully superconducting magnetic system is required to generate a steady toroidal field (TF) of about $5 \mathrm{~T}$ and a time changing poloidal field (PF) of the order of $2 \mathrm{~T}$ at the plasma axis. In addition, the PF system provides the poloidal flux changes necessary to generate and sustain the plasma current.

In the actual coil configuration, these goals are achieved by flowing a steady state current in the TF system, generating a peak field of about $11 \mathrm{~T}$ on the TF magnets, and with a set of preprogrammed, time variable currents in the PF system, generating a peak magnetic field on the PF coils (central solenoid) ranging between +13.5 and $12.5 \mathrm{~T}$, and field changes in the TF system of the order of 2T. The 1 fference operation of the whole system consists of periodical discharges (or plasma burns) and dwell and recharge times (to regenerate the initial condition for the burn) with a nominal minimum period of $500 \mathrm{~s}$.

Furthermore, due to limitations in the understanding of the physical processes in the plasma, there is a possibility that the plasma current suddenly collapses during the burn time. Even if damped by the electromagnetic shielding provided by the conducting structures, the magnetic field in the TF and PF coils will change by about $2 \mathrm{~T}$ in a time scale of $50 \mathrm{~ms}$, with field change rates in the order of $40 \mathrm{~T} / \mathrm{s}$.

From this brief description of the operation of NET it is clear that all the magnets will "see" field changes of remarkable amplitude, and that the effects of these changes will influence the design and operation of the system very strongly. Table I reports the typical design values for the PF and TF systems in term of maximum field, field variation and field change rate in normal operation and during plasma disruptions.

The main consequence of the changing magnetic field on the conductor is the dissipation of energy through AC losses. This thermal input will have a detrimental effect on the temperature and energy margin of the cable as it will increase the cable temperature and decrease the critical current. For fast transients the potential energy deposition is actually in the range of the expected energy margin. Finally, the thermal load of the AC losses must be removed by the refrigerator, thus influencing the size, cost and complexity of the plant. All these three aspects indicate how $\mathrm{AC}$ losses are a factor of merit for superconducting cables for NET. This, however, has to be balanced against other aspects in the cable, such as current distribution, manufacturing complexity, technological feasibility and cost. The prediction of the typical losses expected in the NET coils, together with their influence on the operation and safety margin of the magnets, have been treated elsewhere [2]. Here we present the experimental effort in the characterization of the AC behaviour of the NET $40 \mathrm{kA}$ cable-in-conduit prototype conductor.

\section{CONDUCTOR LAYOUT AND SAMPLES}

The companies ABB (CH) and LMI (I) have been entrusted in 1988 with the development of large current, Nb3Sn wind-and-react conductor [3]. The layouts of the three cable-in-conduit prototype

Manuscript received June 25, 1991. conductors are in Fig. 1. One of the ABB prototypes has braided subcables [4], the other two are purely stranded configurations.

The test concerns the hysteresis losses of the Nb3Sn strands and the coupling losses at different cabling stages up to full size jacketed conductors. We report here the results of the extended test programme carried out at ECN and Twente University (NL). Other AC losses measurements on analogous specimens at PSI $(\mathrm{CH})$ and ENEA (I) basically confirm the loss results reported below $[5,6]$.

The specimens for hysteresis losses are open coils of insulated strands: the volume of the strand specimens, wound as multilayer solenoids on a ceramic former is in the range of $8 \mathrm{~cm}^{3}$. Both LMI and ABB purchased the strand from TWCA. The specifications for the two strands were similar. The layout of the subelements (modified jelly rolls MJR) of the two strands is the same (Nb99Til filaments and $\mathrm{CuSn}: \mathrm{Nb}=1.92$ ) and an identical critical current density has been measured $\left(j_{c} \sim 680 \mathrm{~A} / \mathrm{mm}^{2}\right.$ at $\left.12.5 \mathrm{~T}, 4.2 \mathrm{~K}\right)$. The number and the size of the MJRs assembled in the two strands is different. The LMI strand had 18 MJRs with $\emptyset \sim 60 \mu \mathrm{m}$ and ABB had 54 MJRs with $\emptyset \sim 44 \mu \mathrm{m}$ (Fig. 2). The Cu:non-Cu ratio is 1.25 for both strands, the twist pitch is $10 \mathrm{~mm}$ (ABB) and $12.5 \mathrm{~mm}$ (LMI). The barrier is made by layered vanadium and niobium sheets. The RRR measured on samples reacted after $\mathrm{Cr}$ plating is very poor: $R R R \sim 35$.

The specimens for the coupling current losses are bare sections of the different cable stages, up to $40 \mathrm{~cm}$ long. The specimens of full size cable without jacket have been encased into dummy jackets which have been removed after the heat treatment. To restore the original geometric containment, an insulating foil has been inserted between the cable and dummy jacket. The subcable specimens of the ABB conductors have been inserted into quartz tubes for the heat treatment.The achieved void fraction is larger compared to the full size cable. The subcable specimens of LMI have been inserted into thin steel tube and compacted to the nominal void fraction.

The hysteresis losses have been measured as the area of the magnetization loop. The voltage of a pick-up coils bridge is digitally integrated and the calibration of the loop area is obtained by boil-off calorimetry under continuous sweep operation. The coupling current losses are measured by boil-off calorimetry. The sensitivity of the calorimeter has been improved [7] to be able to measure the coupling losses of the strands (low losses, small volume specimen).

The time varying magnetic field is generated by small solenoid for the strand specimens and by dipoles or race track coils for the cable specimens. The maximum solenoidal dc field for magnetization measurements is $8 \mathrm{~T}$. The maximum $\mathrm{dB} / \mathrm{dt}$ was $-3 \mathrm{~T} / \mathrm{sec}$. For the coupling losses test, a small amplitude, sinusoidal ac field $(+0.025$ $\mathrm{T}$ ) without background dc field was used to reduce the contribution of the hysteresis losses to the total losses.

TABLE 1

TYPICAL DESIGN VALUES FOR THE OPERATION OF THE NET COILS

\begin{tabular}{llllll}
\hline Coil & \multicolumn{3}{l}{ Normal Operation } & \multicolumn{2}{l}{ Plasma Disruption } \\
\hline & $\begin{array}{l}\mathrm{B}_{\max } \\
(\mathrm{T})\end{array}$ & $\begin{array}{l}(\Delta \mathrm{B})_{\max } \\
(\mathrm{T})\end{array}$ & $\begin{array}{l}(\mathrm{dB} / \mathrm{dt})_{\max } \\
(\mathrm{T} / \mathrm{s})\end{array}$ & $\begin{array}{l}(\mathrm{AB})_{\max }(\mathrm{dB} / \mathrm{dt})_{\max } \\
(\mathrm{T} / \mathrm{s})\end{array}$ \\
\hline $\begin{array}{l}\text { P1-P4 } \\
\text { (centr. } \\
\text { solen.) }\end{array}$ & 13.5 & 26.0 & 2.12 & 1 & 15 \\
\hline $\begin{array}{l}\text { P5 } \\
\text { (divert) }\end{array}$ & 11.0 & 11 & 1.26 & 0.1 & 3 \\
\hline $\begin{array}{l}\text { P6-P7 } \\
\text { (equilib.) }\end{array}$ & 5.5 & 5.5 & 0.02 & 0.15 & 2.5 \\
\hline TF & 11.25 & 2 & 0.6 & 2 & 40 \\
\hline
\end{tabular}

0018-9464/92\$03.00 @ 1992 IEEE 

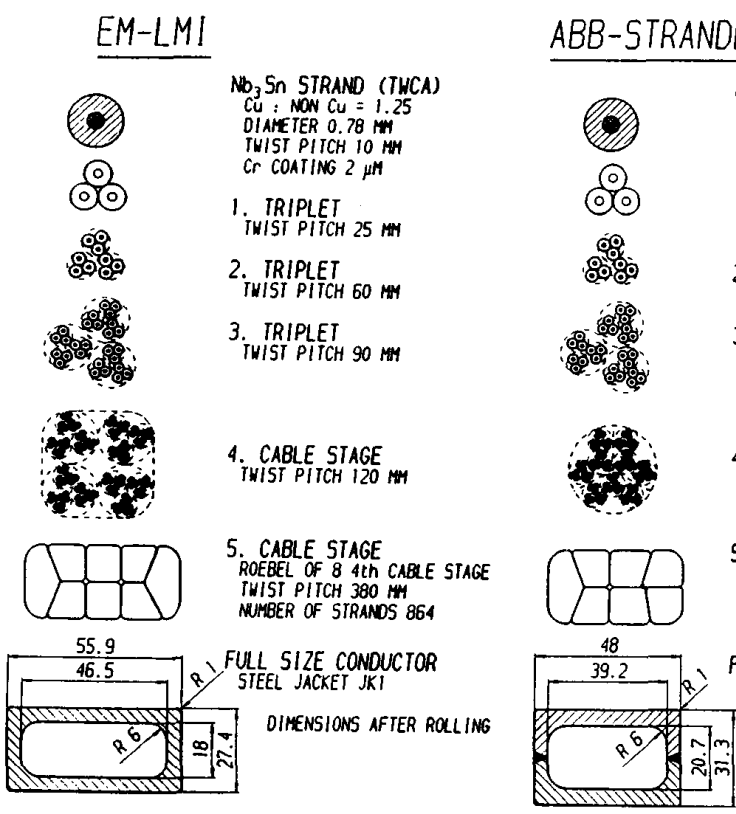

Fig. 1 Layout of the three $40 \mathrm{kA}$ cable-in-conduit superconductors developed for NET

\section{HYSTERESIS LOSSES}

The hysteresis losses per cycle per non-copper strand volume in perpendicular applied field are reported in Fig. 3 as a function of the amplitude of the bipolar field sweep. The hysteresis losses are very large, as it was expected considering that, to achieve the required non-copper critical current density, the spacing between the $\mathrm{Nb}$ filaments in the matrix was kept very low. During the reaction heat treatment, most of the Nb3Sn filaments within each MJR get connected by superconducting bridges. The magnetization current flow over contours which enclose an area much larger compared to the cross section of the individual filaments, and the hysteresis loop is several times larger than expected for a strand with the same number of separated superconducting filaments.

The NET target for the non-copper hysteresis losses for a cycle \pm 3 $T$ is $850 \mathrm{~mJ} / \mathrm{cm}^{3}$, which is about one half of the measured values. On the other hand, these strands represent the technology status in 1988. Since then the strand manufactures (including TWCA) have achieved a substantial reduction of the hysteresis losses (less bridging between the $\mathrm{Nb}_{3} \mathrm{Sn}$ filaments), keeping the critical current density within the NET requirement, $\mathrm{jc} \geq 620 \mathrm{~A} / \mathrm{mm}^{2}$ at $12.5 \mathrm{~T}, 4.2 \mathrm{~K}$.

Besides the measurements of the $+3 \mathrm{~T}$ hysteresis loss, which was an acceptance test, an interesting result is found by an analysis of the hysteresis losses in superimposed ac + dc field. It is known [8] that the same small $\Delta \mathrm{B}$ variation produces different losses on a
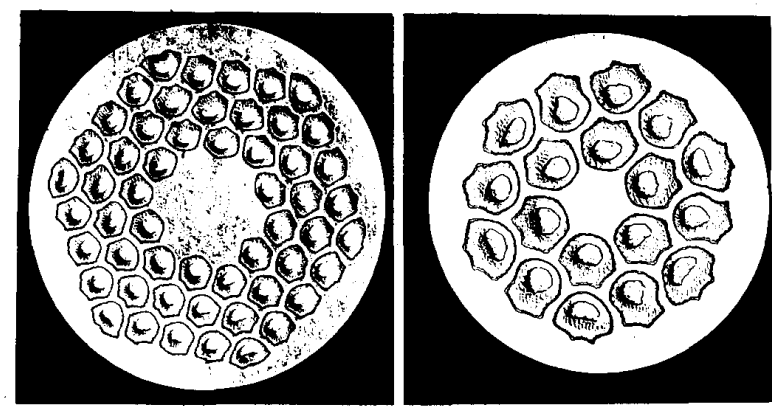

Fig. 2 Cross section of the modified jelly roll strand supplied by TWCA to ABB (left) and LMI (right)

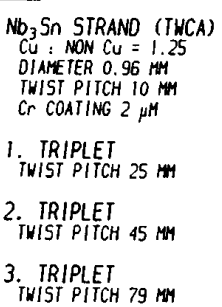

4. CABLE STAGE

5. CABLE STAGE

ROEBEL OF 7 TRIPLETS

THIST PITCH 359 MM

FULL SIZE CONOUCTOR

STEEL JACKET AISI 316LN

2 LONGITUOINAL LASER WELOS
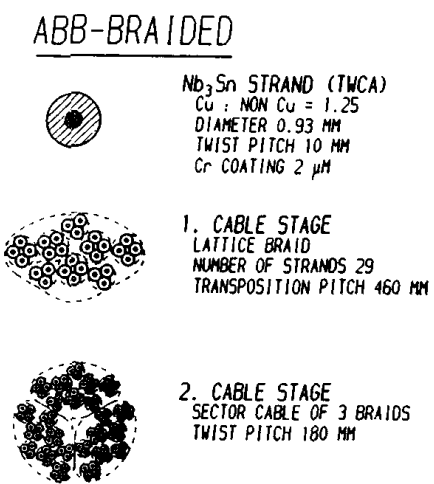

2. CABLE STAGE 3 BRAIOS

TWIST PITCH 180 MM

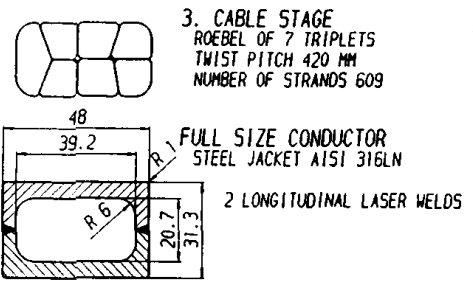

filamentary superconductor depending on the background dc field, i.e. depending on the penetration field $B p$ : this can be written as $q=f$ $\left(\triangle B, B p(B)\right.$ ). As function of $B p$ (or $j_{c}$ ) the hysteresis losses per cycle per unit volume of superconductor, $q$, get a maximum when $B p$ $=\Delta B / 2$ : this maximum is no longer a function of $B p$, but only depends on $\Delta B$, i.e. $q_{\max }=f(\Delta B)$. This maximum is the same, provided that the basic geometry of the filaments is the same, i.e. all the round filament strands will have the same $q_{\max }$ independent of the diameter size, which only influences the background field corresponding to the loss maximum. The condition to observe the maximum is that the applied $\Delta \mathrm{B}$ is smaller than twice the first penetration field.

Even for hollows cylinder filaments, the loss curve of superimposed ac $+\mathrm{dc}$ field has a maximum whose amplitude depends only on $\Delta \mathrm{B}$. In this last case $\mathrm{q}_{\max }$ is higher (up to $20 \%$ ) than calculated for round filaments [9]. Looking at the experimental results in Fig. 4, ( $\left(\mathrm{B}_{\mathrm{dc}}\right)$ for $\left.\Delta \mathrm{B}=0.2,0.4,0.6 \mathrm{~T}\right)$, we observe that the measured $\mathrm{qmax}_{\text {max }}$ is always higher than the expected value for round and hollow cylinder. The shape of the integration domaines in the bridged structure of the MJR is responsible for the larger $\mathrm{q}_{\max }$. Even assuming an arbitrary filament diameter, it is not possible for superimposed dc + ac fields to reduce the loss behaviour of an interconnected, "bridged" geometry to that of a round filament.

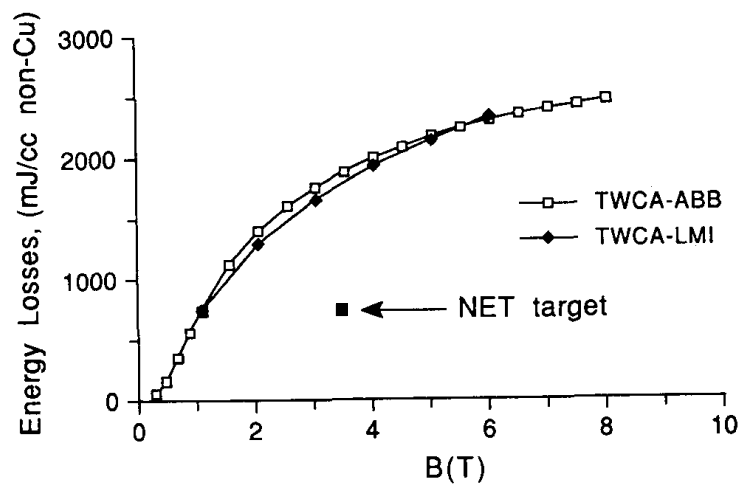

Fig. 3 Hysteresis losses per non-copper volume in perpendicular applied field for bipolar cycles $+B$ on the two TWCA strands 


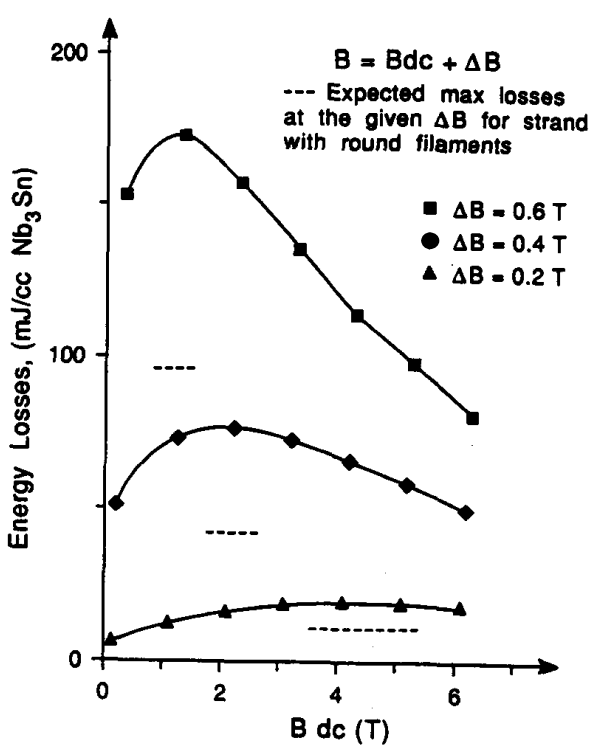

Fig. 4 Hysteresis losses per Nb3Sn volume in superimposed ac + dc field as a function of the background de field

Of course it would be possible to draw some formula fitting the experimental results, but its validity range would be practically limited to the examined strand. Another strand with bridged filaments but different geometric layout (spacing and grouping of filaments) will need different fitting parameters.

Although the "effective" filament diameter, deff should not be arbitrarily used to calculate the hysteresis losses in any field scenario, it gives a measure of the size of the bridging between the filaments. From the measured penetration field, $\mathrm{Bp}$, at $6 \mathrm{~T}$ and the corresponding Jc (only $\mathrm{Nb}_{3} \mathrm{Sn}$ cross section), using the critical state formula

$$
\mathrm{deff}_{\mathrm{ef}}=\frac{\mathrm{Bp} \pi}{\mu_{\mathrm{O}} \mathrm{Jc}}
$$

we get $\mathrm{d}_{\mathrm{eff}}=54 \mu \mathrm{m}(\mathrm{LMI})$ and $\mathrm{d}_{\mathrm{eff}}=38 \mu \mathrm{m}$ (ABB). This result is in good agreement with the size of the MJRs in the two strands.

\section{COUPLING CURRENT LOSSES}

The coupling current losses in transverse field are proportional to $(\mathrm{dB} / \mathrm{dt})^{2}$. Our characterization criteria consists of finding out experimentally the proportionality constant, $n \tau$, as input for the computing code: $n \tau$ is defined by the loss formula for ramped or sinusoidal field

$$
p=\frac{n \tau}{\mu_{0}}(d B / d t)^{2} \quad p=\frac{B o^{2} 2 \pi^{2} n \tau f^{2}}{\mu_{0}}
$$

where $p$ is the power dissipated per strand unit volume (jacket material and $\mathrm{He}$ cross section are not taken into account).

Applying a sinusoidally pulsed field, $B=B_{0} \sin 2 \pi f t$, the energy losses per cycle, q, are plotted against the frequency of the field pulse. At low frequency $(f<0.03 / \tau) q$ is a linear function at the frequency:

$$
q=a_{0}+a_{1} f
$$

From the experimental slope of the loss curve a1 $\left[\mathrm{J} / \mathrm{m}^{3} \mathrm{~Hz}\right]$, we get

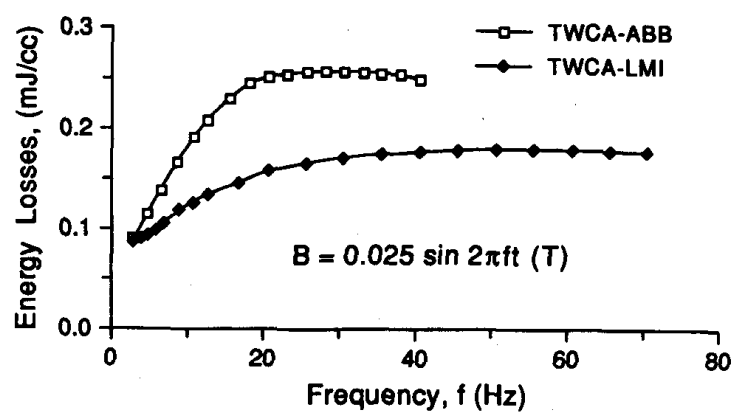

Fig. 5 Coupling current losses per strand volume of the individual strands

$$
\mathrm{n} \tau=\frac{\mathrm{a}_{1} \mu_{\mathrm{O}}}{2 \pi^{2} \mathrm{~B}_{\mathrm{o}}^{2}}=\frac{\mathrm{a}_{1} 2 \cdot 10^{-7}}{\pi \mathrm{B}_{\mathrm{o}}^{2}}
$$

In a multistage conductor with non insulated subcables, the largest contribution to the transverse coupling current losses is given by the last cable stage. In the loss curve of the full size conductor, the measured slope corresponds to the largest $n \tau$, i.e. the above mentioned proportionality constant. The geometry factor, $\mathrm{n}$ is included in the experimental slope: $n=2$ only in case of round composite with homogeneously distributed filaments, what is never the case of the large multistage $40 \mathrm{kA}$ conductors. In case of a rectangular cable cross section, $\mathrm{n} \tau$ is different for the two orientations of the field and both values of $n \tau$ have to be measured. The minimum length of the specimens is one length of the twist pitch of the last cable stage.

\section{A. Individual Strands}

From the slopes of the curves in Fig. 5, we estimate $n \tau=0.6 \mathrm{~ms}$ (for TWCA-LMI strand) and $\mathrm{n} \tau=1.4 \mathrm{~ms}$ (for TWCA-ABB strand). The graphical evaluation error is less than $10 \%$.

The range of the results is in good agreement with the expected values, but the large difference between the two strands is surprising, considering that the two layouts are very similar and that the higher losses are observed in the strand with shorter twist pitch. Actually the intrastrand coupling current losses are not a relevant parameter for the computation of the ac losses in the NET coils. The crucial parameter for the transverse coupling losses is the $n \tau$ value of the full size conductor, which takes into account all the coupling current loops, but is dominated by the interstrand coupling currents of the last cable stage.

\section{B. Subcables}

The larger $\mathrm{n} \tau$ measured on the LMI specimens can be understood taking into account the larger void fraction of the ABB samples (encased in quartz glass tubes) and the conducting steel jacket of the LMI samples. The average transverse resistivity of the subcable samples is then reduced in the LMI samples.

It is interesting to observe how the $\mathrm{n} \tau$ grows with increasing subcable size, i.e. twist pitch. A soldered cable or a monolithic conductor without resistance barriers with the same twist pitch as, for example, the ABB-87 $(180 \mathrm{~mm})$, can approach $n \tau$ values of several hundreds msec. The limited number of strand crossovers and their high contact resistance due to the chrome plating increase the mean transverse resistance by almost two orders of magnitude.

TABLE II

$n \tau$ MEASUREMENTS ON SUBCABLE SAMPLES [msec]

\begin{tabular}{ll}
\hline ABB-27 twist & 3.4 \\
ABB-29 braid & 2.5 \\
ABB-87 3x braid & 7.3 \\
LMI-27 twist & 6.2 \\
LMI-108 twist & 8.6
\end{tabular}


TABLE III

COUPLING CURRENT LOSSES ON FULL SIZE SAMPLES

\begin{tabular}{lllll} 
Conductor & Jacket & Field & Lab & m $[\mathrm{msec}]$ \\
\hline ABB-T & yes & $\|$ & ECN & 24.2 \\
ABB-T & yes & $\|$ & TU & 56.7 \\
ABB-T & no & $\|$ & ECN & 18.3 \\
ABB-T & no & $\|$ & TU & 16.1 \\
ABB-T & no & 1 & TU & 36.1 \\
ABB- & yes & $\|$ & ECN & 88.7 \\
ABB-L & yes & $\|$ & TU & 75.3 \\
ABB-L & no & $\|$ & ECN & 18.3 \\
LMI & yes & $\|$ & ECN & 15.3 \\
LMI & no & $\|$ & ECN & 14.4 \\
LMI & yes & 1 & ECN & 91.4 \\
LMI & no & $\perp$ & ECN & 9.5
\end{tabular}

\section{Full Size Conductor}

For each conductor, a specimen with jacket and another without jacket was measured. The pulsed field was applied parallel to the broad side of the rectangular conductor. This field component is by far the most relevant in the sections of the central solenoid where the conductor has the lowest temperature margin. The losses in the field direction perpendicular to the broad side have been also measured in most of the specimens. The slope of the curves in the linear range is often difficult to estimate because of the scattering of the points, which is much larger compared to the strand and subcable specimens. The graphical error for the estimation of $n \tau$ is between 10 and $20 \%$. Another unsatisfactory aspect of the loss curves is that the extrapolation to 0-frequency (i.e. the hysteresis loss components) is much different from one conductor to the other, even when they are manufactured from the same strand. The loss curves are shown in Fig. 6 and 7 and the results on the full size sample test are summarized in Table III. The electrical contact with the jacket is specified, the direction of the pulsed field is parallel to the broad side (II) or perpendicular to the broad side ( $\perp$ ).

All the samples, independent of the field direction, have significantly higher losses when cable and jacket are in electrical contact, suggesting that a part of the coupling current induced in the cable flow back through the jacket. On the other hand, it cannot be excluded that the large difference between jacketed and non-jacketed samples is due to a manipulation problem: the crossover resistance is eventually changed, i.e. increased, when the cable has been extracted from the jacket. The contribution of the steel jacket to the AC losses has been measured separately on a hollow jacket specimen of the same length as the superconducting specimens. Eddy current and hysteresis losses of the jacket are definitely negligible with respect to the full size conductor samples.

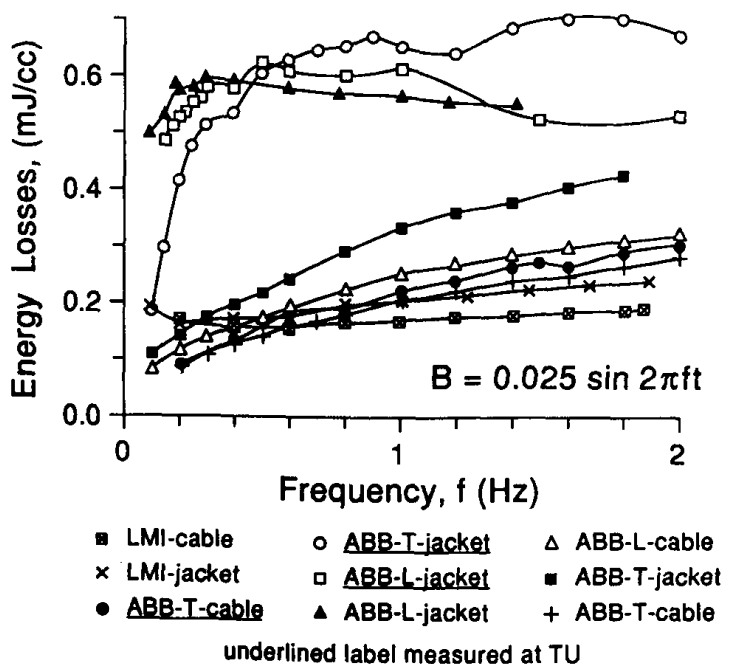

Fig.6 Coupling current losses of full size conductor specimens with field perpendicular to the short side

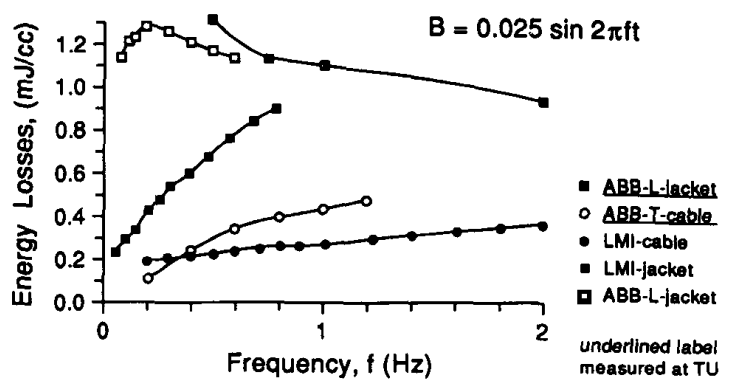

Fig. 7 Coupling current losses of full size conductor specimens with field perpendicular to the broad side

The higher losses of the sample ABB-T jacket compared to the LMI-jacket, which has exactly the same cabling pattern, suggest that the transversal resistance is proportional to the number of strand crossovers, in this case to the number of strands.

\section{CONCLUSION}

From the point of view of the $A C$ losses, the three conductors developed at $\mathrm{ABB}$ and LMI have very similar behaviour. The hysteresis losses are much larger compared to the NET specification, but new strands developed in the meantime fulfill the NET requirement on both the hysteresis losses and critical current density.

The coupling current losses of the individual strands are kept at a satisfactory low value $(\tau<1 \mathrm{msec})$. Thanks to the high contact resistance of the $\mathrm{Cr}$ plating, about one hundred strands can be bundled together keeping $\tau$ lower than 4 msec.

The losses with the field perpendicular to the broad side of the rectangular conductor are always the largest. The ratio of the slopes of the losses in the two field orientations, $(n \tau) \perp /(n \tau)$ ) is of the order of 1.5 to 6 , depending on the aspect ratio and on the steel jacket. According to the coil geometry and current scenario, it should be assessed what is the maximum acceptable $(n \tau) \perp /(n \tau))_{\|}$to guarantee that the stability margin in the low field sections, with large field component perpendicular to the broad conductor side, does not get lower than the design stability margin for the coil sections with the highest field and orientation parallel to the broad side.

The $n \tau$ values of the full size jacketed conductors are in the range of the design value of NET for two out of three samples. A further reduction of the coupling current losses will be achieved in future by limiting the final twist pitch to values $<350 \mathrm{~mm}$.

\section{REFERENCES}

(1) E. Salpietro, et al., Fusion Technology, 14, No. 1, 1988

[2] L. Bottura, U. Mszanowski, N. Mitchell 'Analysis of the ITER superconducting coils: magnetic field, ac losses and cooling", published in this volume.

[3] J.V. Minervini et al., 'Progress in the development of a $40 \mathrm{kA}$ superconductor for the NET central solenoid', proc. of MT-11 Conf., pp. 880-885, Tsukuba, Japan 1989 .

[4] P. Bruzzone 'Fully transposed braids for the cable-in-conduit superconductors of NET', published in this volume.

5] K. Kwasnitza, Ch. Widmer 'Final report of NET contract NET/88-317 on magnetization and ac losses measurements', PSI Villigen, March 90.

[6] R. Bruzzese, S. Chiarelli, P. Gislon, M. Spadoni 'Final report of NET contract NET/88-190 on subcomponent test for a NET OH conductor', ENEA Frascati, January 91.

[7] J.A. Eikelboom An apparatus for calorimetric measurement of ac losses in superconductors' Cryogenics 31, 363-365 (1991)

[8] K. Kwasnitza, P. Bruzzone 'Hysteresis losses of multifilament superconductors in superimposed dc and ac field', Cryogenics 21, 593-597 (1981)

[9] J.A. Eikelboom, R.A. Hartmann, L.J.M van de Klundert 'Hysteresis losses in hollow superconductors', IEEE Trans. Magn. Vol. 25, pp. 1968-71, March 1989. 